\title{
A Case of Anterior Choroidal Artery Occlusion and Stroke Secondary to External Compression
}

\author{
Satya Narayana Patro ${ }^{a, b} \quad$ Khawaja Hassan Haroon ${ }^{a, b}$ \\ Khansabegum Tambolia Abdulaziz Zafar ${ }^{b, c}$ Suhail Hussain ${ }^{a}$ \\ Ahmad Muhammada, b \\ ${ }^{a}$ Neuroscience Institute, Hamad Medical Corporation, Doha, Qatar; ${ }^{b}$ Weill Cornell Medicine- \\ Qatar, Doha, Qatar; 'Internal Medicine, Hamad Medical Corporation, Doha, Qatar
}

Keywords

Anterior · Choroidal artery · Occlusion · Stroke

\begin{abstract}
The anterior choroidal artery ( $\mathrm{AChA}$ ) is a small artery commonly arising from the supraclinoid segment of the internal carotid artery (ICA). The significance of the AChA is related to its strategic supply to various important structures of the brain, such as the optic tract, the posterior limb of the internal capsule, the cerebral peduncle, the lateral geniculate body, medial temporal lobe, medial area of pallidum, and the choroid plexus [J Neurol. 1988;235:387-91]. The AChA syndrome in its complete form consists of the triad of hemiplegia, hemisensory loss, and hemianopia. However, incomplete forms are more frequent in clinical practice [Stroke. 1994;25:837-42]. Isolated infarction in the AChA territory is relatively rare. The presumed pathogenic mechanisms of AChA infarction are cardiac emboli, large-vessel atherosclerosis, dissection of the ICA, small-vessel occlusion, or other determined or undetermined causes [Stroke. 1994;25:837-42 and J Neurol Sci. 2009;281:80-4].
\end{abstract}

\section{Introduction}

Here, we report a case of isolated anterior choroidal artery (AChA) infarction secondary to compression of the artery caused by a hemorrhagic lesion in the left choroidal fissure, most likely representing a small acute hemorrhage due to ruptured cavernoma. 

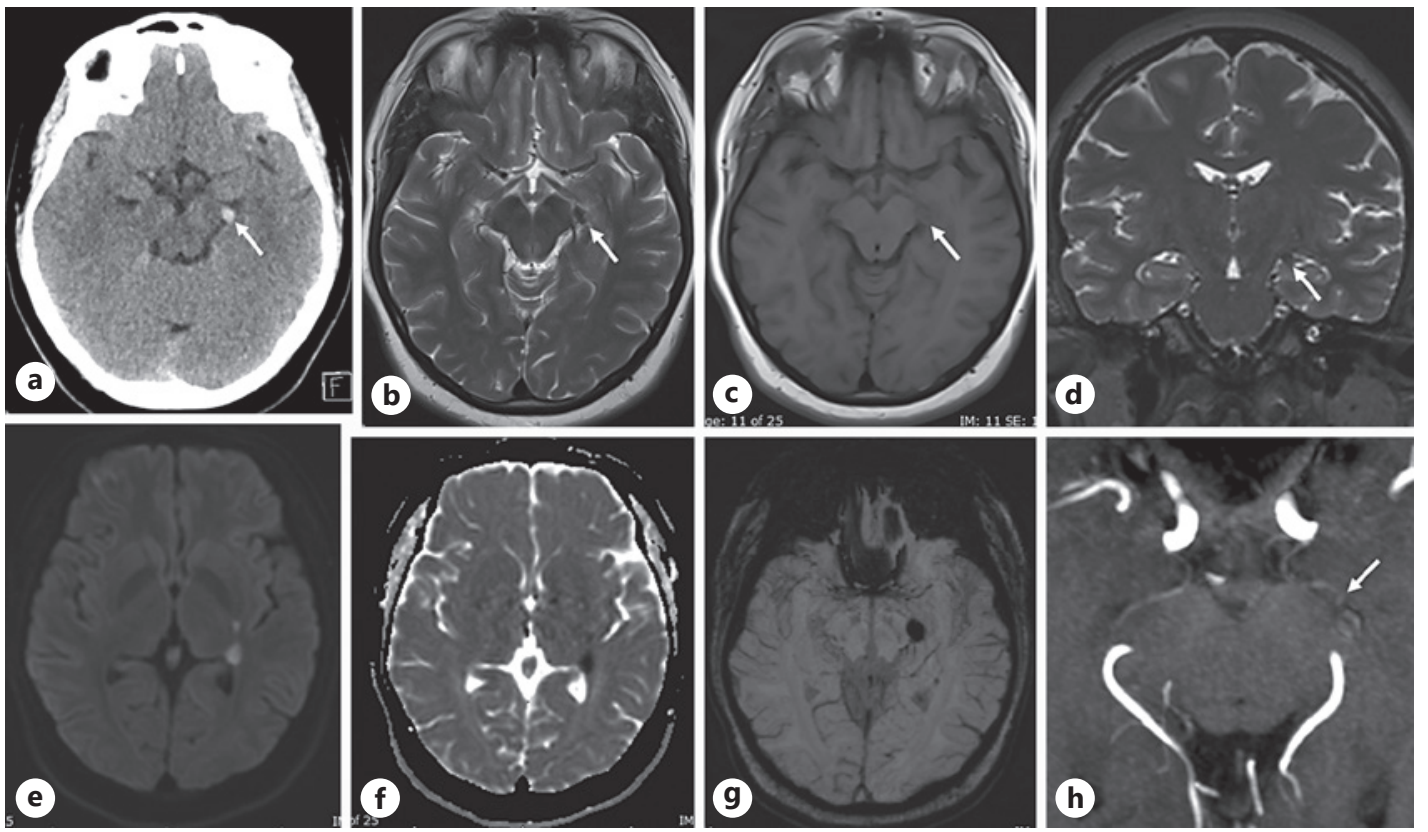

Fig. 1. a CT head axial section demonstrated a small hyperdense lesion in the left choroid fissure (white arrow). b-d Axial T2W, axial T1W, and coronal T2W images showed small acute hemorrhagic lesion in the left choroid fissure (white arrow). e, f Axial DWI and corresponding ADC map showed acute infarction in the left AChA territory. $\mathbf{g}$ Axial SWI image demonstrated a small blooming lesion adjacent to the left cerebral peduncle. $\mathbf{h}$ Axial time of flight image showed the small left AChA compressed by the hemorrhagic lesion. AChA, anterior choroidal artery.

\section{Case}

A 41-year-old female schoolteacher diabetic on oral hypoglycemic and hypertensive without any medication presented with left hemicranial headache which built up gradually over 2 days. The headache was moderate in intensity and associated with nausea and visual disturbance in her right eye since the morning of presentation. Her initial neurological examination was suggestive of right upper motor neuron facial palsy, along with right pronator drift and right homonymous hemianopia. Her National Institute of Health Stroke Scale (NIHSS) was 4 . Her initial blood pressure was $161 / 120 \mathrm{~mm} \mathrm{Hg}$, which dropped to 142/80 after $1 \mathrm{~h}$ without any intervention. CT stroke protocol (CT head plain, CT angiogram, and CT perfusion) showed a small hyperdense hemorrhagic lesion in the left choroid fissure, while her angiogram and CT perfusion were unremarkable (Fig. 1). The hyperdense lesion was considered as a focal bleed, and intravenous tPA was not administered. The possibilities considered were hypertension, micro-AVM, or cavernoma with bleed or a thrombosed aneurysm. The next day, the patient underwent MRI/MRA of the head and neck, which revealed acute infarction in the left AChA territory involving the left hippocampus, left posterior putamen, posterior limb of the internal capsule, posterolateral thalamus, and tail of caudate. The intracranial MRA showed abrupt occlusion of the faintly visible left AChA by the small hemorrhagic lesion in the left choroid fissure (Fig. 1). Subsequently, the patient underwent digital subtraction angiography, which did not reveal any aneurysms or vascular malformations. However, the left AChA was abruptly cut off in the left choroidal fissure (Fig. 2). The conclusion drawn from all the imaging was that of a hemorrhagic lesion in the left choroid fissure, compressing the left AChA and resulting in acute infarction in its 

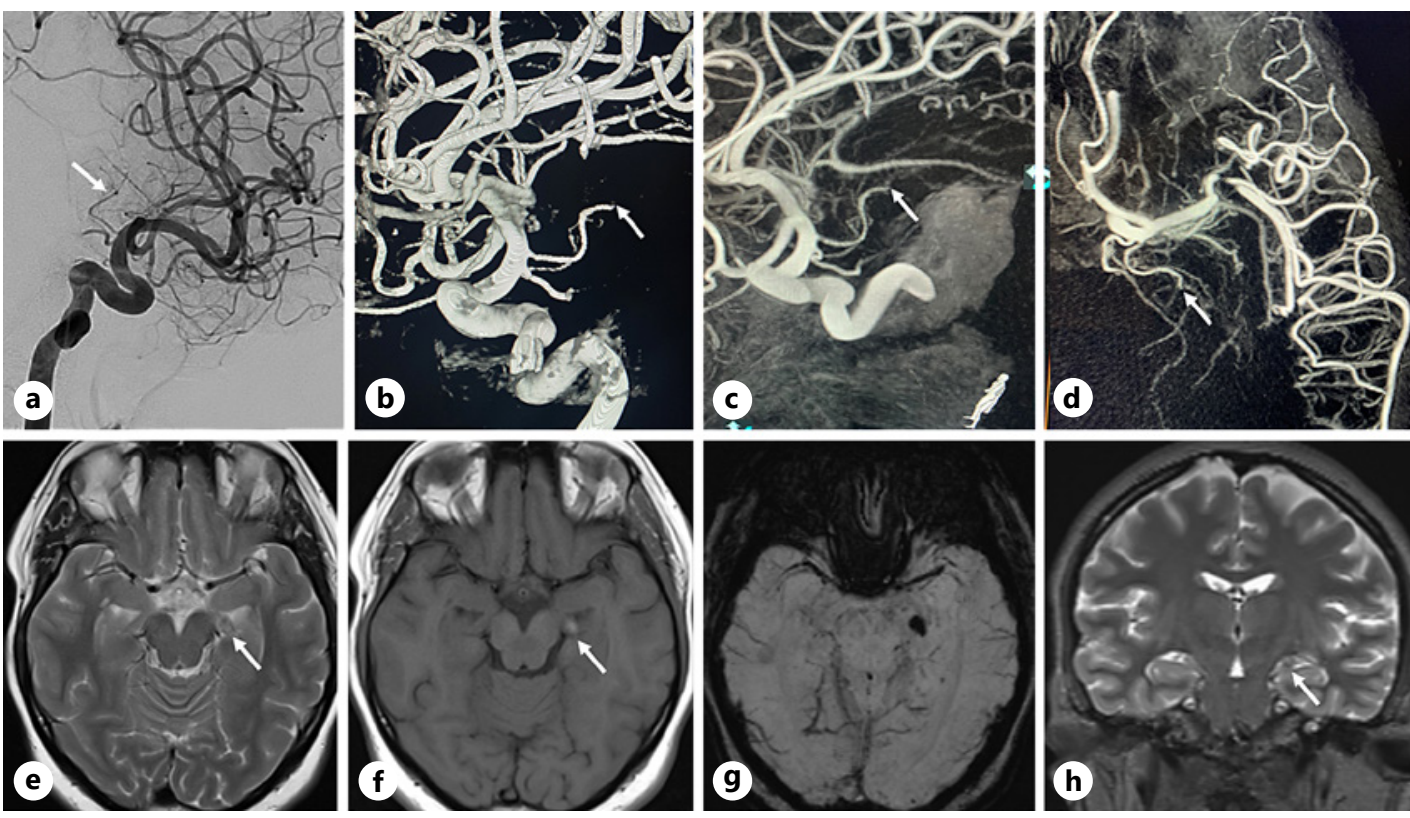

Fig. 2. a Digital subtraction angiogram image demonstrated abrupt occlusion of the left AChA. b-d 3D reconstruction and Xper CT reconstruction showed abrupt occlusion of the left AChA in the left choroid fissure region (white arrow). e-h Axial T2W, T1W, SWI, and coronal T2W images showed stable hemorrhagic lesion in the left choroid fissure (white arrow). AChA, anterior choroidal artery.

territory. The patient was treated conservatively, and she showed improvement during her hospital stay. There was no family history of intracranial hemorrhage. The patient was discharged after 5 days with minimal residual deficit. She underwent a follow-up MRI after a month, which revealed stable hemorrhagic lesion in the left choroidal fissure with no new infarction.

Her renal functions, lipid profile, echocardiogram, and fundus examination did not show any evidence of end-organ damage. The 48-h in patient cardiac monitoring did not reveal evidence of paroxysmal AF.

\section{Discussion}

Although the AChA is a thin artery, it supplies extremely important regions of the brain such as the optic tract, the posterior limb of the internal capsule, the cerebral peduncle, the lateral geniculate body, medial temporal lobe, medial area of pallidum and the choroid plexus [1]. The AChA commonly originates from the posterior wall of the internal carotid artery in $96-99.5 \%$ of cases, $2-5 \mathrm{~mm}$ distal to the posterior communicating artery and 2-5 $\mathrm{mm}$ proximal to the carotid bifurcation. Occasionally, the AChA has been found to arise from the intracerebral carotid bifurcation or from the PComA [2]. The AChA is mostly found as a single branch (84.6\%); however, it can be a double $(13 \%)$ or a triple branch $(2.4 \%)$ at its origin.

The course of the AChA can be divided into 2 segments: the cisternal and the plexal, and the entry point of the AChA into the lateral ventricle at the choroid fissure is described as the plexal point [3]. The AChA may have short and hypoplastic or long and hyperplastic course. The long course type usually has anastomoses with the lateral posterior choroidal 
artery, the posterior cerebral artery, and the posterior communicating artery [4]. Even if the AChA exhibits this collateral circulation, the AChA is highly vulnerable, and infarction in its territory can be devastating [5]. The extent of the AChA territory is still debatable. The territory can be divided into deep or perforating, which includes the posterior twothirds of the internal capsule, the adjacent optic and auditory radiations, the medial portion of the globus pallidus, the tail of the caudate nucleus, and the posterior paraventricular part of the corona radiata. The superficial artery territory includes the amygdala, the uncus, the head of the hippocampus, the piriform cortex, and the lateral aspect of the lateral geniculate body [6].

The AChA is only visible between 71 and $98 \%$ on conventional angiogram due to its small diameter or because it is obscured by overlapping vessels [7]. On lateral angiograms, the plexal point is at $18-26 \mathrm{~mm}$ from the origin of the AChA. MRI using 3D-TOF MR angiography, particularly on $3 \mathrm{~T}$ scanner, allows identification of the AChA in $95 \%$ of cases, due to the higher signal of the arteries and better spatial resolution compared to the $1.5 \mathrm{~T}$ scanner. The anatomic features that help identify the artery on the MR angiographic source images are (a) its typical point of origin from the posterior wall of the internal carotid artery, and (b) its characteristic posterior course along the medial aspect of the temporal lobe through the ambient cistern to the choroidal fissure.

The AChA is involved in many diseases, including infarction, aneurysm, Moyamoya disease, brain AVM/AV fistula, and tumor. Therefore, it is an important artery supplying vital structures of the brain.

The prevalence of isolated AChA infarcts is low, ranging from 2.9 to $11 \%$ [8]. The AChA syndrome in its complete form consists of the triad of hemiplegia, hemisensory loss, and hemianopia. However, incomplete forms are more frequent in clinical practice [9]. The most constant neurological deficit in AChA territory infarcts is hemiplegia, which is caused by the involvement of the pyramidal tract in the posterior limb of the internal capsule. Hemisensory loss, due to the involvement of the ventral posterolateral nucleus of the thalamus or of the thalamocortical fibres, is less frequent. Homonymous hemianopia caused by the involvement of the lateral geniculate body or of the geniculocalcarine tract is rare. Hemineglect in rightsided infarcts and mild aphasia in left-sided infarcts are other usual findings. Acute pseudobulbar mutism, amnesia, and pseudobulbar palsy can also be seen due to unilateral or bilateral AChA territory infarcts.

The AChA infarcts have been considered as lacunar infarcts because of the small artery diameter with predominant motor impairment. However, other causes such as large-vessel disease, cardioembolic, or other determined or undetermined causes that have been considered too [9, 10]. Ois et al. [10] studied AChA infarction in a prospective series of 1,350 patients with acute ischemic stroke and found that compared to hemispheric infarcts, AChA stroke patients were younger, more often male and diabetic, and less likely to be pretreated with antithrombotic therapy than hemispheric stroke patients. The same authors observed that AChA infarcts were less associated with embolic sources and showed a higher risk of progression but a lower risk of recurrence or mortality than hemispheric infarcts [10]. Recent studies also commented on the prognostic factors in AChA infarcts. Larger infarcts particularly $>20 \mathrm{~mm}$ or with superficial territory involvement had worse prognosis $[6,10]$.

Although rare, a possible mechanism of cerebral infarction is mechanical compression of the cerebral artery by a neighboring lesion. A neoplasm or aneurysm may cause infarction. Meningiomas and other tumors have been reported to cause stenosis or occlusion of the intracranial arteries, leading to ischemic events [11]. There is 1 case report of posterior communicating artery aneurysm causing compression of AChA [12]. In the present case, the left AChA was occluded by mechanical compression from an adjacent hemorrhagic lesion in the left choroid fissure.

\section{Karger'}




\section{Conclusion}

The compressing hemorrhagic lesion in our case remained stable over 1 month. Most likely, the lesion represents an initially ruptured small cavernoma, given the stable size over a period of 1 month. To our knowledge, this is the first case of a cavernoma causing adjacent arterial occlusion by compression mechanism. Our case suggests that evaluation for a compressive lesion should be considered in certain stroke patients. This mechanism of ischemic stroke has been described rarely in the literature.

\section{Statement of Ethics}

This article was conducted ethically in accordance with the World Medical Association Declaration of Helsinki. The subject in this paper gave her written informed consent to publish her case including publication of images. Written informed consent for publication was obtained from the patient.

\section{Conflict of Interest Statement}

The authors have no conflicts of interest to disclose.

\section{Funding Sources}

The authors declare that they did not receive any funding.

\section{Author Contributions}

Dr. Satya Narayana Patro: manuscript development, patient management, and image review. Dr. Khawaja Hassan Haroon: manuscript development, patient management, literature review, and critical review. Dr. Khansabegum Tamboli: patient management and literature review. Dr. Abdulaziz Zafar: literature review and critical review. Dr. Suhail Hussain: literature review and critical review. Dr. Ahmad Muhammad: manuscript development, patient management, literature review, and critical review.

\section{References}

1 Helgason CM. A new view of anterior choroidal artery territory infarction. J Neurol. 1988;235:387-91.

2 Morandi X, Brassier G, Darnault P, Mercier P, Scarabin JM, Duval JM. Microsurgical anatomy of the anterior choroidal artery. Surg Radiol Anat. 1996;18:275-80.

3 Rhoton AL Jr, Fujii K, Fradd B. Microsurgical anatomy of the anterior choroidal artery. Surg Neurol. 1979;12: 171-87.

4 Abrahams JM, Hurst RW, Bagley LJ, Zager EL. Anterior choroidal artery supply to the posterior cerebral artery distribution: embryological basis and clinical implications. Neurosurgery. 1999;44:1308-14.

5 Choi $\mathrm{CY}$, Lee $\mathrm{CH}$. Transposition of anterior choroidal artery and posterior communicating artery origin. J Korean Neurosurg Soc. 2012;52:240-2.

6 Sohn H, Kang DW, Kwon SU, Kim JS. Anterior choroidal artery territory infarction: lesions confined to versus beyond the internal capsule. Cerebrovasc Dis. 2013;35:228-34.

7 Takahashi S, Suga T, Kawata Y, Sakamoto K. Anterior choroidal artery: angiographic analysis of variations and anomalies. AJNR Am J Neuroradiol. 1990;11:719-29.

\section{Karger's}


8 Hupperts RM, Lodder J, Heuts-van Raak EP, Kessels F. Infarcts in the anterior choroidal artery territory. Anatomical distribution, clinical syndromes, presumed pathogenesis and early outcome. Brain. 1994; 117(Pt 4):825-34.

9 Leys D, Mounier-Vehier F, Lavenu I, Rondepierre P, Pruvo JP. Anterior choroidal artery territory infarcts. Study of presumed mechanisms. Stroke. 1994;25:837-42.

10 Ois A, Cuadrado-Godia E, Solano A, Perich-Alsina X, Roquer J. Acute ischemic stroke in anterior choroidal artery territory. J Neurol Sci. 2009;281:80-4.

11 Launay M, Fredy D, Merland JJ, Bories J. Narrowing and occlusion of arteries by intracranial tumors. Review of the literature and report of 25 cases. Neuroradiology. 1977;14:117-26.

12 Lim SW, Chang CH, Yeh CH, Chio CC, Chen CW. Cerebral infarction due to anterior choroidal artery occlusion caused by posterior communicating artery aneurysm compression. Formos J Surg. 2012;45(2):51-4. 